\title{
Brain-derived Neurotrophic Factor and Neurotrophin-3 Activate Striatal Dopamine and Serotonin Metabolism and Related Behaviors: Interactions with Amphetamine
}

\author{
Mathew T. Martin-Iverson, ${ }^{1}$ Kathryn G. Todd, ${ }^{1}$ and C. Anthony Altar ${ }^{2}$ \\ ${ }^{1}$ Neurochemical Research Unit, Department of Psychiatry, Walter Mackenzie Health Sciences Centre, University of \\ Alberta, Edmonton, Alberta, Canada T6G 2B7 and 'Regeneron Pharmaceuticals Inc., Tarrytown, New York 10591
}

To investigate behavioral and neurochemical effects of neurotrophic factors in vivo, rats received continuous $14 \mathrm{~d}$ infusions of either brain-derived neurotrophic factor (BDNF), neurotrophin-3 (NT-3), or vehicle unilaterally into the substantia nigra. BDNF and NT-3 decreased body weights, an effect that was sustained over the infusion period. BDNF elevated daytime and nocturnal locomotion compared with infusions of vehicle or NT-3. At 2 weeks, a systemic injection of amphetamine $(1.5 \mathrm{mg} / \mathrm{kg}$, s.c.) increased the frequencies and durations of rotations contraversive to the side of BDNF and NT-3 infusions. Both factors attenuated amphetamineinduced locomotion without affecting amphetamine-induced stereotyped behaviors such as sniffing, head movements, and snout contact with cage surfaces. Only BDNF induced backward walking, and this response was augmented by amphetamine. BDNF, but not NT-3, increased dopamine turnover in the striatum ipsilateral to the infusion relative to the contralateral striatum. Both trophic factors decreased dopamine turnover in the infused substantia nigra relative to the contralateral hemisphere and increased 5-HT turnover in the striatum of both sides. Contraversive rotations were positively correlated with dopamine content decreases and 5-HT turnover increases in the striatum ipsilateral to the infused substantia nigra. Backward walking was positively correlated with increased dopamine and 5-HT turnover in the striatum of the infused hemisphere. Supranigral infusions of BDNF and NT-3 alter circadian rhythms, spontaneous motor activity, body weights, and amphetamine-induced behaviors including locomotion and contraversive rotations. These behavioral effects of the neurotrophins are consistent with a concomitant activation of dopamine and 5-HT systems in vivo.

[Key words: brain-derived neurotrophic factor, neurotrophin-3, amphetamine, circadian rhythms, rotational behavior, dopamine, 5-HT]

\footnotetext{
Received May 13, 1993; revised Aug. 5, 1993; accepted Aug. 12, 1993.

We gratefully acknowledge the AMGEN-Regeneron partnership for the supply of BDNF and NT-3, and the Alberta Heritage Foundation for Medical Research and the Alberta Mental Health Resedrch Fund for financial support. Brenda Vos, Lynn Y. Burger, and Richard Strel provided excellent technical assistance for this project.

Correspondence should be addressed to Mathew T. Martin-Iverson at the above address.
}

Copyright (C) 1994 Society for Neuroscience $0270-6474 / 94 / 141262-09 \$ 05.00 / 0$
NGF promotes the survival and differentiation of cultured central cholinergic neurons, and prevents their atrophy following axotomy in vivo (Hefti, 1986). However, NGF does not exhibit these properties on nigral dopamine (DA) neurons (Hefti, 1986; Hartikka and Hefti, 1988; Hyman et al., 1991). Because DA neurons degenerate in Parkinson's disease, other trophic factors have been studied for their ability to prevent the atrophy or promote the survival and growth of these neurons. One such factor is a member of the neurotrophin family of NGFs. This protein, brain-derived neurotrophic factor (BDNF), is structurally related to NGF (Leibrock et al., 1989), and enhances the in vitro survival of cultured cholinergic neurons (Alderson et al., 1990) and dopaminergic neurons (Hyman et al., 1991; Knüsel, 1991). Neurotrophin-3 (NT-3), like BDNF, is also a member of the NGF family of neurotrophins (Maisonpierre et al., 1990; Rosenthal et al., 1990). All three neurotrophic factors share approximately 50\% homology in their amino acid sequence (Leibrock et al., 1989), yet they also show distinct neuronal specificities. Unlike NGF, BDNF and NT-3 promote the survival of nodose ganglion neurons (Lindsay et al., 1985) and dopaminergic neurons (Hyman et al., 1991; Spina et al., 1992) whereas sympathetic neurons primarily respond to NGF, but not to BDNF or NT-3 (Lindsay et al., 1985; Maisonpierre et al., 1990).

The significance of these in vitro actions of BDNF has only recently been extended to in vivo efficacy models. Altar et al. (1992) showed that a 2 week unilateral infusion of BDNF into the neostriatum or the pars compacta of the substantia nigra (SN) of otherwise intact rats induces contraversive rotations (away from the infused hemisphere) subsequent to $d$-amphetamine challenge. More pronounced effects were observed after infusion into the nigra than into the striatum. Additionally, increases in the ratio of DA metabolites to DA levels were observed in the striatum on the side receiving BDNF infusions, suggesting an increase in DA turnover. These findings suggest that BDNF may have a beneficial role in Parkinson's disease, although other neurotransmitter systems may be altered by BDNF infusions in vivo. For example, BDNF-treated rats also exhibit a decrease in body weight to approximately $85 \%$ of their preexperimental weight (Altar et al., 1992). This observation indicates that BDNF may have behavioral effects on its own, in the absence of amphetamine. The reduction in body weight may be the result of behavioral effects similar to those of amphetamine, which is well known to produce anorexia. On the other hand, it is also possible that the reduction in body weight 
was a function of some physiological process affccted by intracranial infusions of BDNF, and behavioral effects of BDNF may be secondary to this decrease in body weight.

The purposes of this study were (1) to attempt to replicate the effect of BDNF on body weights and on amphetamineinduced turning with a lower dose of amphetamine, and to determine if NT -3 could produce similar effects; $(2)$ to ascertain whether the behavioral effects of the neurotrophic factors are secondary to effects on body weights; (3) to determine whether striatal, mesolimbic, or nigral levels of monoamines after amphetamine injections are altered by prior treatment with either trophic factor; and (4) to determine if either BDNF or NT-3 can affect spontaneous behaviors, alter circadian rhythms in locomotor activity, or enhance amphetamine-induced behaviors such as locomotion and stereotypy.

\section{Materials and Methods}

Animals and surgery. Male Sprague-Dawley rats (240-290 gm; $n=7$ $13 /$ group) were housed individually and maintained for $15 \mathrm{~d}$ on a 12 $\mathrm{hr}: 12 \mathrm{hr}$ light/dark cycle with access to food and water. Animal housing consisted of stainless steel cages $(24 \times 16 \times 26 \mathrm{~cm})$ with one Plexiglas side and a wire-mesh floor. The cages were arranged in four rows of 12 boxes on a cage rack. Preexperimental weights were recorded, and the animals randomly assigned to one of the following treatment groups: nonsurgical controls, vehicle controls, NT-3, or BDNF. Rats were anesthetized with Nembutal $(60 \mathrm{mg} / \mathrm{kg}$, i.p. $)$ and mounted in stereotaxic frames in a "flat skull plane" (i.e., tooth bar set at $-3.3 \mathrm{~mm}$ ). Alzet 2002 osmotic minipumps (Alza Corp., Palo Alto, CA) were filled the day before with vehicle (sterile PBS), NT-3 $(1.0 \mu \mathrm{g} / \mu \mathrm{l})$, or BDNF $(1.0$ $\mu \mathrm{g} / \mu \mathrm{l}$ ) and fitted with a $2 \mathrm{~cm}$ piece of silated PE50 tubing (MicroRenathane, Braintree Scientific, Braintree, MA) attached to a $6.8-\mathrm{mm}$ long cannula (Plastics One, Inc., Roanoke, VA). The pumps were implanted subcutaneously through a $2-\mathrm{cm}$-long incision in the scalp. A $0.5-\mathrm{mm}$-diameter hole was drilled in the skull above the right substantia nigra (interaural coordinates: anterior $2.5 \mathrm{~mm}$, lateral $2.7 \mathrm{~mm}$, and ventral $6.8 \mathrm{~mm}$ ); the cannula was inscrted and attached to the skull with cyanoacrylate adhesive, and the skull incision was closed with wound clips. The stability and delivery of BDNF from osmotic pumps maintained at $37^{\circ} \mathrm{C}$ have been estimated at $72-85 \%$ (Altar et al., 1992), and similar values have been obtained for NT-3 (C. A. Altar, unpublished observations).

Animals were weighed daily with the exception of postsurgery days 1,8 , and 15 . Four days after surgery, the vehicle-infused animals were given restricted access to food in order that their weights matched those of the BDNF and NT-3 treated groups. Therefore, the rat body weights were analyzed by ANOVA to ensure that significant group differences would not occur between the vehicle group and the drug groups by restricting the laboratory rat chow for the vehicle group to $15-25 \mathrm{gm}$ each day, as needed. All other groups, including the untreated controls, had ad libitum access to food. All groups had ad libitum access to water.

On postsurgery day 15 , the animals were challenged with $1.5 \mathrm{mg} / \mathrm{kg}$ $d$-amphetamine sulfate (s.c.). The rats were videotaped for $5 \mathrm{hr}, 5 \mathrm{~min}$ every hour for $2 \mathrm{hr}$ before amphetamine and for $3 \mathrm{hr}$ after amphetamine. Each rat was killed by decapitation $4 \mathrm{hr}$ after amphetamine injection. All procedures involving live animals were approved by the Health Sciences Animal Welfare Committee of the University of Alberta to meet with Canadian Council on Animal Care guidelines.

Behavioral analysis. Locomotor counts were obtained for the duration of the experiment by computer recording of infrared photobeam interruptions in the rats' home cages. Video cameras mounted on moveable camera trucks recorded the animals' behaviors on day 15 for $295 \mathrm{sec}$ at hourly intervals twice before amphetamine injections and three times after injections (see Martin-Iverson, 1991, for specific details of the apparatus and proccdurcs). Vidcotapcs were later rated by a trained observer blind to the drug conditions of the animals and to the purposes of the experiment, using a computer behavioral observation program (BEBOP) that records both frequencies and durations of each of 30 different behaviors. These behaviors included clockwise (ipsiversive) turns (right turns defined as a change in the orientation of the trunk of at least $90^{\circ}$ ) and counterclockwise (contraversive) rotations (left turns), backward walking, and sniffing.
Histology and neurochemical analysis. Brains were removed and inspected for the site of the termination of the cannula tract. The left and right striatum, nucleus accumbens, and substantia nigra were dissected out on ice, rapidly frozen with dry ice, and frozen at $-80^{\circ} \mathrm{C}$ for future analysis. HPLC with electrochemical detection was used to determine levels of the catecholamines and their major metabolites (Baker et al., 1987). For the substantia nigra (SN), tissue was sometimes pooled across two different animals from the same group (i.e., the right or left $\mathrm{SN}$ of two animals were sometimes pooled together).

Statistical analysis. Body weight data were subjected to analysis of variance (ANOVA) with one between factor (infusion treatment) and one within factor (days of treatment). Photobeam interruptions during the 2 week treatment period were summed in $12 \mathrm{hr}$ blocks and analyzed similarly with ANOVA, except that there were two within factors (14 consecutive $24 \mathrm{hr}$ periods and day/night). If there are more than two repeated measures with this type of data, a major assumption of ANO$V A$ is violated (i.e., homogeneity of variances and covariances) leading to inaccurate estimations of probability (Vitaliano, 1982). Therefore, a number of different multivariate methods (Pillais Trace, Hotellings $T$, and Wilks Lambda) were used in all of these cases as part of the statistical software (SPSSPC). In situations in which the multivariate methods and the ANOVA are in agreement, only the ANOVA results are shown. In other situations, the multivariate statistics are taken as the most valid.

Periodograms were made for each rat from the photobeam interruption counts (consecutive blocks of $1 \mathrm{hr}$ ), which involve plotting power (the sum of the squared amplitudes of the sinusoids determined by fast Fourier analysis) versus the frequency of the sinusoid (cycles/hour, STATGRAPHICS software). Integrated periodograms were also plotted, comparing the average normalized periodograms of each group to the $95 \%$ confidence interval of the Kolmogorov-Smirnoff bounds around a random uniform distribution of the cumulated sum of the squared amplitudes of the sinusoids. On the basis of these periodograms, it was clear that all four groups exhibited statistically significant $24 \mathrm{hr}$ rhythms in photobeam interruptions, but the amplitudes at all other cycles were within the $95 \%$ confidence interval for random amplitudes. Therefore, cosinor functions were made for circadian $(24 \mathrm{hr})$ rhythms following the procedure described by Halberg et al. (1972). Three parameters of the circadian rhythms were obtained: the acrophase [the time (in radians) of the peak of the sinusoid function], the mesor [the average hourly photobeam interruptions for each rat (in the present case with equal intervals of measurements)], and the amplitude (the difference between the level of activity at the acrophase and at the mesor). These three measures were statistically analyzed by ANOVA with one between factor (drug treatment). Most of the behavioral measures taken just prior to and after amphetamine injections were analyzed by ANOVA with one between factor (drug treatment) and one within factor (time). In the case of rotations, a number of rats from the untreated control and vehicle-infused control groups displayed few rotations, if any. Since this makes assessment of variability difficult, rotations in both directions were summed for the two preamphetamine observation periods and the three postamphetamine observation periods, and these sums for each group were analyzed by the nonparametric Mann-Whitney $U$ test (onetailed test corrected for ties) comparing contraversive with ipsiversive turns, with the expectation of greater contraversive turning. All comparisons between groups for data subjected to ANOVA were carried out with the multiple $F$ test for planned comparisons or Tukey's HSD test for post hoc individual comparisons (Kicss, 1989).

\section{Results}

Cannula placements. All of the cannulas were observed to terminate in the area (zona incerta) just above the $\mathrm{SN}$, as previously described with this procedure (Altar et al., 1992). One animal exhibited a blood clot in the $\mathrm{SN}$ and was excluded from further analysis.

Body weights. The mean weights of each treatment group are represented over time in Figure 1 . There was a significant treatment $\times$ day interaction for body weight $[F(33,407)=11.6 ; p$ $<0.001]$. Individual comparisons indicated that there were no significant differences between rats treated with BDNF or NT-3 and the food-deprived vehicle controls over the experimental period. The nonsurgical control group maintained a significantly higher body weight than the treated groups after day 4 (Fig. 1). 


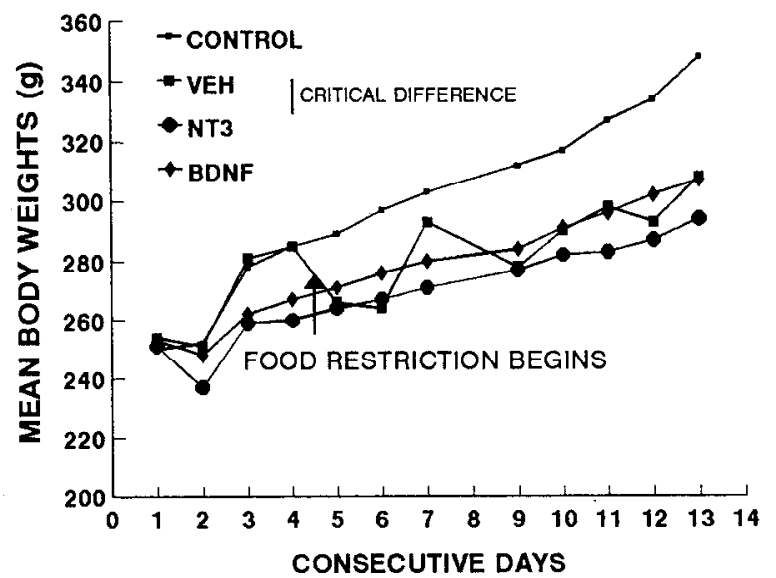

Figure 1. Mean body weights of rats that were untreated (CONTROI), or that received infusions of vehicle $(V E H)$, BDNF, or NT-3 for 2 weeks after surgery. The critical difference line indicates the degree of difference required between any two groups for statistical significance, $p<0.05$ (multiple $F$ test). On day 4 and thereafter, rats in the vehicle group had food access restricted to $15-20 \mathrm{gm} / \mathrm{d}$ in order for their weights to match those of rats in the BDNF and NT-3 groups.

Motor activity. Figure 2 shows the mean photobeam interruptions over consecutive days and nights. There was a significant treatment $\times$ day $\times$ day/night interaction $[F(39,481)=$ $2.95 ; p<0.001]$. As can be seen in Figure $2 A$, the BDNFtreated group exhibited significantly higher levels of daytime locomotion than the vehicle-infused and NT-3-infused groups over most days.

Locomotor activity was higher in the BDNF-treated group than in the untreated, freely feeding group on 13 of $14 \mathrm{~d}$, but this increase was rarely statistically significant. A similar general relationship can be observed for nocturnal locomotion (Fig. 2B). The RDNF-infused group exhibited significantly greater activity levels than either the vehicle or the NT-3-infused groups. BDNF increased activity significantly above the untreated controls on days $2-4$, but not on any other nights. The nocturnal but not daytime activity of the vehicle-infused controls increased significantly above all other groups on the two nights following food restriction. Food restriction had no effect during the day, when rats do not normally spend much time eating, and the increase in nocturnal activity returned to normal levels or lower, indicating habituation to the food restriction regimen.

Periodograms of the photobeam interruptions (Fig. 3) show that the greatest amplitude of sinusoids occurred in all four groups at $24 \mathrm{hr}(0.0416 \mathrm{cycles} / \mathrm{hr})$. Further ultradian rhythms can be observed at 12,8 , and $6 \mathrm{hr}$, and to a lesser degree at a fcw shorter periods, but all of the amplitudes of these sinusoids fell within the $95 \%$ Kolmogorov-Smirnov bounds for a uniform distribution. These data indicate that rats in all four groups exhibited significant circadian rhythms in motor activity, and so these rhythms were subjected to Halberg's cosinor procedure. The cosinor functions of the $24 \mathrm{hr}$ rhythms for all four groups (Fig. 4) were generated from the mean values for the mesors, amplitudes, and acrophases (Table 1), and ANOVA revealed significant treatment effects on mesors $[F(3,38)=6.0 ; p<$ $0.005]$, amplitudes $[F(3,38)=2.9 ; p<0.05]$, and acrophases $[F(3,38)=4.9 ; p<0.01]$. The BDNF group had a higher mesor (mean level of activity) and a later acrophase (time of peak of the cosinor function) than either the vehicle-treated or the NT3-treated groups. The circadian rhythm parameters for the BDNF
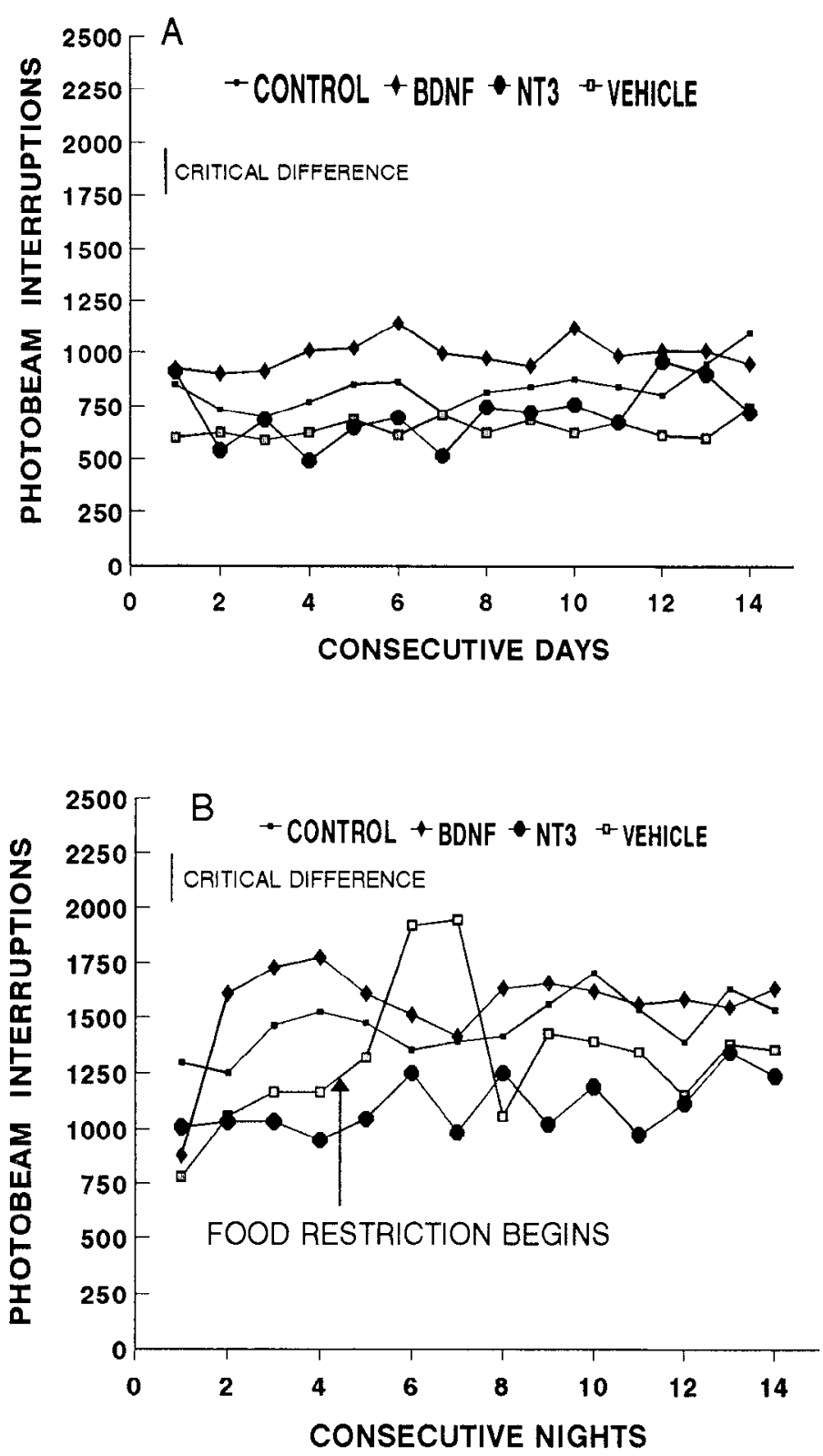

Figure 2. Mean photobeam interruptions summed over the $12 \mathrm{hr}$ light $(A)$ or dark $(B)$ portions of the days in the groups that were either untreated $(C O N T R O L)$ or treated with vehicle, BDNF, or NT-3. The critical difference lines indicate the degree of difference required between any two groups for statistical significance, $p<0.05$ (multiple $F$ test).

group were similar to those of the untreated, freely feeding controls. The most striking alteration in circadian rhythms was that NT-3 reduced the amplitudes of the circadian rhythm (Figs. 3, 4), and this reduction was statistically significant relative to all other treatment groups. NT-3 also shifted the timing of the peak of the rhythm earlier in the night (to 01:48), compared with the BDNF and vehicle groups. The amplitude of the circadian rhythm in motor activity of the vehicle-infused animals did not differ from that of either the untreated controls or the BDNF groups. However, vehicle infusions shifted the acrophases $(01: 00)$ to a greater extent than did NT-3 relative to both untreated controls and BDNF-treated animals.

Amphetamine-induced rotations. Most groups did not exhibit differences between the duration or frequency of contraversive and ipsiversive rotations as a total from the two $5 \mathrm{~min}$ obser- 


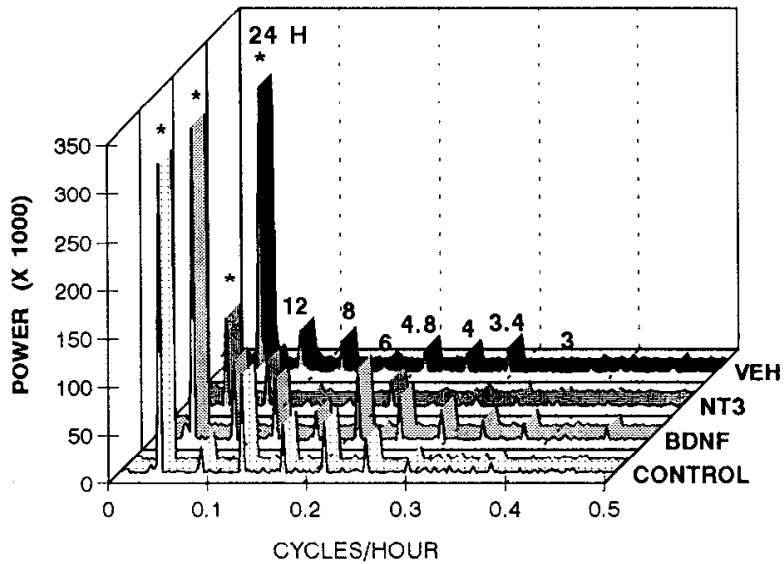

Figure 3. Periodograms showing the sum of the squared amplitudes of the sinusoids (power) at various frequencies (periods of the sinusoids expressed as cycles per hour) for each of the groups. Numbers above the peaks represent the length of the periods in hours. The figure clearly demonstrates that the strongest rhythms occur with periods of $24 \mathrm{hr}$ (circadian), with relatively minor rhythms at shorter periods. *, significantly different from amplitudes expected by chancc, given a continuous distribution $(p<0.05$, Kolmogorov-Smirnoff test).

vation periods prior to amphetamine injections (Fig. 5). The exception was that the NT-3 group exhibited a low but significantly greater median duration of contraversive rotations than ipsiversive rotations (Mann-Whitney $U=19 ; p<0.025$ ). Both NT-3 and BDNF infusions greatly elevated the duration (NT3: $U=21, n=9, p<0.05$; BDNF: $U=52, n=14, p<0.025$ ) and frequency (NT-3: $U=22, p<0.05$; BDNF: $U=50, p<$ 0.02 ) of contraversive turning as a sum of the three $5 \mathrm{~min}$ observation periods after amphetamine. In contrast, the untreated controls and vehicle-treated groups exhibited a greater frequency and duration of ipsiversive than contraversive turning after amphetamine, but these differences were not statistically significant.

Amphetamine-induced motor activity. Photobeam interruptions for $1 \mathrm{hr}$ prior to amphetamine injections and for $4 \mathrm{hr}$ after amphetamine revealed a treatment $\times$ hour interaction $[F(12$, $152)=3.7 ; p<0.001]$. As shown in Figure 6, there were no

Table 1. Effects of no treatment (control), or 2 weeks of continuous unilateral infusions of vehicle, BDNF, or NT-3 into the SN, on circadian rhythms in motor activity

\begin{tabular}{lcll} 
Treatment & Mesor \pm SEM & Amplitude \pm SEM SEM (min)] & $\begin{array}{c}\text { Acrophase } \\
{[\mathrm{hr}: \min \pm}\end{array}$ \\
\hline Control & $94 \pm 10$ & $41.2 \pm 4.6$ & $02: 30 \pm 15$ \\
Vehicle & $80 \pm 4$ & $39.9 \pm 4.2$ & $01: 00 \pm 17 \dagger$ \\
BDNF & $105 \pm 6^{*}$ & $42.0 \pm 4.5$ & $03: 00 \pm 21^{* *}$ \\
NT-3 & $73 \pm 5 \dagger$ & $25.6 \pm 2.9 \ddagger$ & $01: 48 \pm 45$
\end{tabular}

Values were obtained by counting photobeam interruptions in blocks of $1 \mathrm{hr}$. The mesor was obtained by calculating the average hourly activity over the 2 weeks of treatment. The amplitude (difference of peak and mesor) and the acrophase (time of the peak) were obtained from fast Fourier transforms (see Materials and Methods) of the photobeam interruption data. Statistical analysis of the acrophases was conducted on the values as radians.

* Significantly different from vehicle ( $p<0.05$, multiple $F$ test).

** Significantly different from vehicle and NT-3 $(p<0.05$, multiple $F$ test).

$\dagger$ Significantly different from control $(p<0.05$, multiple $F$ test).

$¥$ Significantly different from all other treatment groups $(p<0.05$, multiple $F$ test).

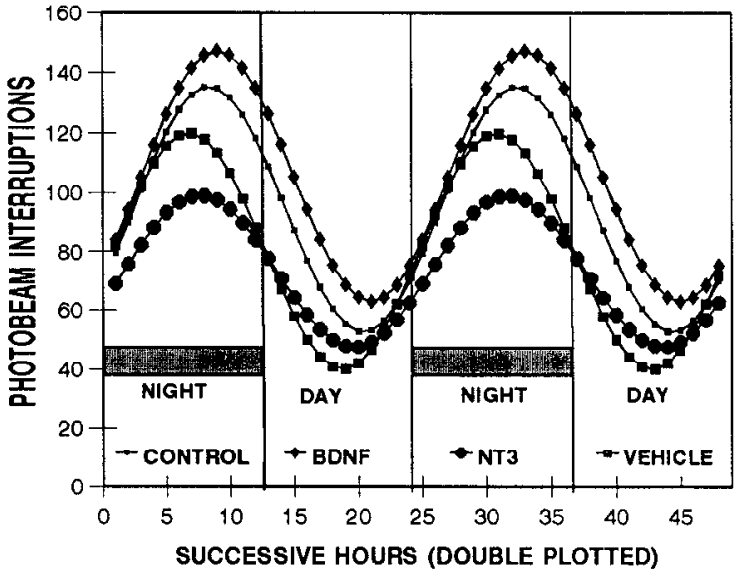

Figure 4. Graphic representation of cosinor functions of circadian rhythms from the data reported in Table 1 . These plots represent estimated photobeam interruptions from amplitudes, acrophases, and mesors of sinusoids with a period of $24 \mathrm{hr}$.

differences between groups in the $1 \mathrm{hr}$ prior to amphetamine injections. Photobeam interruptions (locomotor activity) were increased by amphetamine, and amphetamine-induced motor activity was lower in NT-3-treated and BDNF-treated rats. No significant differences were observed between untreated controls and vehicle-infused animals.

Amphetamine-induced backward walking. There were significant treatment effects for both the duration $[F(3,38)=11.8$; $p<0.001]$ and frequency $[F(3,38)=10.9 ; p<0.001]$ of backward walking. Multivariatc tests also indicatcd significant treatment $\times$ hour interactions for both of these measures [e.g., Hotellings $T$ (approximate): $F(12,101)=5.2, p<0.001$ (duration); $F(12,101)=4.1, p<0.001$ (frequency)]. As can be seen in Figure 7, BDNF increased backward walking whereas none of the other groups did, and the BDNF effect was increased by amphetamine.

Other behaviors. A number of behaviors were altered by amphetamine, but did not exhibit differences between the treatment groups. Behaviors increased by amphetamine but not differently affected by the treatments included sniffing, head movements, standing, and snout contact with a surface of the cage. Behaviors uniformly decreased by amphetamine in all treatment groups were lying down and sleeping.

Neurochemical analyses. The effects of the treatments on striatal levels of DA, 3,4-dihydroxyphenylacetic acid (DOPAC), homovanillic acid (HVA), 5-HT, and 5- hydroxyindoleacetic acid (5-HIAA) at $4 \mathrm{hr}$ after amphetamine injections are shown in Table 2. ANOVA revealed a significant overall treatment $x$ side of brain effects only for 5-HIAA treatment $[F(3,37)=7.3$; $p<0.002]$. There was significant main effect of drug on DOPAC levels $[F(3,37)=3.21 ; p<0.05]$. However, a number of the individual comparisons between specific groups were significant (Table 2). These include increases ipsilateral to the side of infusion in DOPAC after BDNF and in HVA after BDNF and NT-3 relative to untreated controls. 5-HIAA was significantly increased by both BDNF and NT-3 in both right and left striata relative to untreated controls and vehicle infusions (Table 2), and BDNF increased 5-HIAA levels significantly more in the right striatum (ipsilateral to the infused $\mathrm{SN}$ ) compared to the left. No significant neurochemical changes of any kind were observed in the nucleus accumbens (data not shown). 

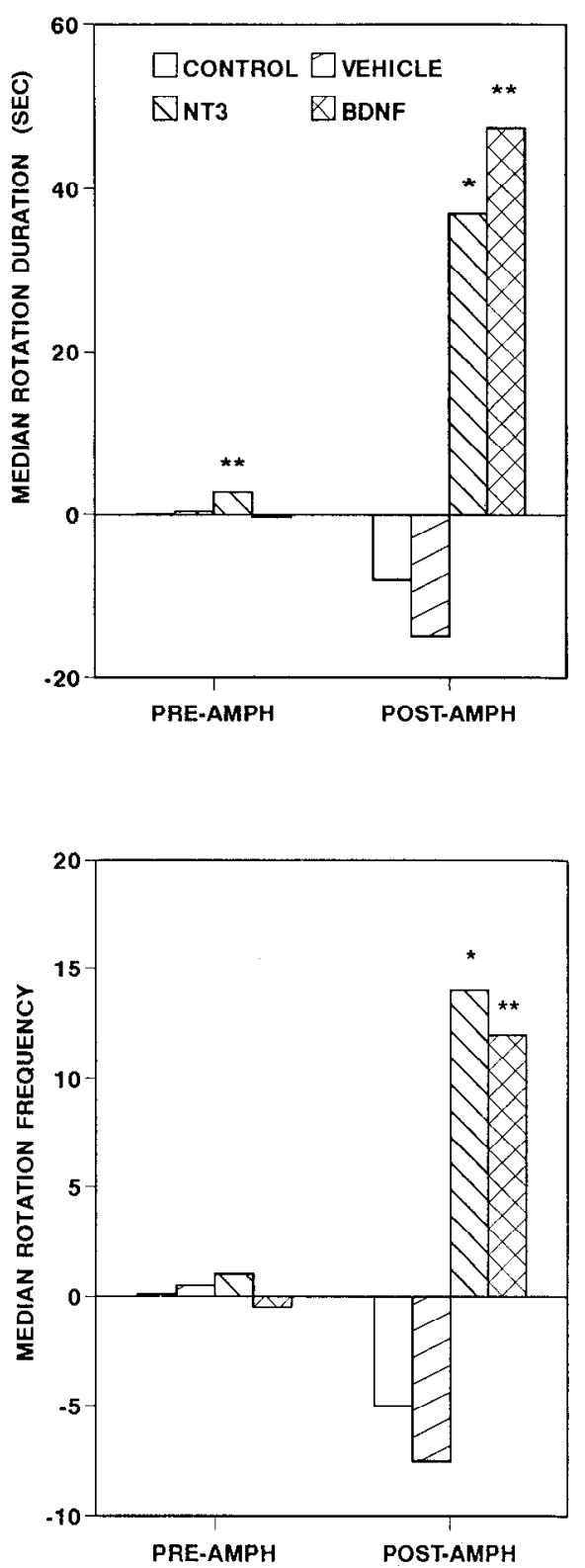

Figure 5. Median durations (top) and frequencies (bottom) of the net contraversive rotations (contraversive minus ipsiversive rotations) preand postamphetamine $(A M P H ; 1.5 \mathrm{mg} / \mathrm{kg}$, s.c.) challenge. Net contraversive turns are shown above zero, and net ipsiversive turns are displayed as below zero. Contraversive rotations significantly different from ipsiversive rotations: ${ }^{*}, p<0.05 ;^{* *}, p<0.01$ (Mann-Whitney $U$ test)

Analysis of DA turnover, measured by the ratio (DOPAC + HVA):DA from each right striatum as a percentage of the ratio from the left striatum, showed a significant treatment effect $[F(3$ $37)=4.1 ; p<0.02$ ]. Individual comparisons indicated that only BDNF increased DA turnover in the operated right striatum relative to the noninfused left striatum (Fig. 8). Statistical analysis of DOPAC:DA ratios from the SN (HVA levels were too low for reliable measurement in this brain region) as a percentage of the ratios from the left striatum revealed a significant treatment effect $[F(3,18)=3.5 ; p<0.05$; Fig. 8]. Subsequent comparisons showed that BDNF and NT-3 significantly decreased DA turnover in the right $\mathrm{SN}$ versus the left. A similar ratio analysis of 5-HT turnover (5-HIAA:5-HT) indicated that

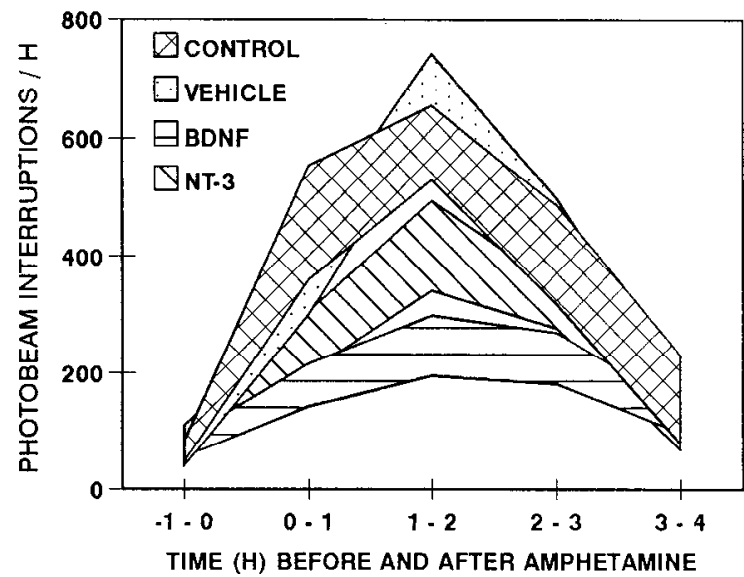

Figure 6. The SE range of photobeam interruptions around the mean $1 \mathrm{hr}$ before and $4 \mathrm{hr}$ after amphetamine $(1.5 \mathrm{mg} / \mathrm{kg})$ injections. Nonoverlapping areas are significantly different from each other $(p<0.05$, multiple $F$ test).

there were no significant unilateral effects of the infusions (data not shown).

There was a significant correlation found between decreases in levels of DA in the right striatum and net contraversive rotations $5 \mathrm{~min}$ before death and $4 \mathrm{hr}$ after amphetamine injections (correlation coefficient $=-0.327 ; p<0.03$ ). Table 3 shows the significant correlations between backward walking and neurochemical measures from the striatum. Both durations and frequencies of backward walking positively correlated with

Table 2. Effects of no treatment (control, $n=7)$, or vehicle $(n=11)$, BDNF $(n=13)$, or NT-3 $(n=9)$ infusions into the right SN, on striatal monoamine levels ( $\mathrm{ng} / \mathrm{gm}$ tissue) as determined by HPLC on triplicates (see Materials and Methods for details), $4 \mathrm{hr}$ after amphetamine injection $(1.5 \mathrm{mg} / \mathrm{kg}$, s.c.)

\begin{tabular}{llcc}
$\begin{array}{l}\text { Neurochemical } \\
\text { measure }\end{array}$ & Treatment & $\begin{array}{c}\text { Right striatum } \\
\text { (mean } \pm \text { SEM) }\end{array}$ & $\begin{array}{c}\text { Left striatum } \\
\text { (mean } \pm \text { SEM) }\end{array}$ \\
\hline Dopamine & Control & $4741 \pm 356$ & $4876 \pm 270$ \\
& Vehicle & $5125 \pm 207$ & $5297 \pm 264$ \\
& BDNF & $4665 \pm 210$ & $5375 \pm 308$ \\
& NT-3 & $4735 \pm 275$ & $4625 \pm 275$ \\
DOPAC & Control & $853 \pm 70$ & $943 \pm 70$ \\
& Vehicle & $983 \pm 48$ & $1041 \pm 47$ \\
& BDNF & $1230 \pm 103^{* *}$ & $1163 \pm 66$ \\
HVA & NT-3 & $1088 \pm 81$ & $1176 \pm 131$ \\
& Control & $354 \pm 22$ & $372 \pm 21$ \\
& Vehicle & $431 \pm 16$ & $454 \pm 23$ \\
& BDNF & $479 \pm 34^{* *}$ & $443 \pm 29$ \\
5-HT & NT-3 & $470 \pm 43^{* *}$ & $424 \pm 49$ \\
& Control & $353 \pm 21$ & $358 \pm 52$ \\
& Vehicle & $360 \pm 18$ & $401 \pm 55$ \\
& BDNF & $456 \pm 97$ & $335 \pm 17$ \\
5-IIIAA & NT-3 & $405 \pm 47$ & $496 \pm 129$ \\
& Control & $390 \pm 26$ & $393 \pm 32$ \\
& Vehicle & $368 \pm 14$ & $372 \pm 15$ \\
& BDNF & $513+29 *+* *$ & $447 \pm 25 \dagger^{* *}$ \\
& NT-3 & $475 \pm 28 \dagger^{* *}$ & $460 \pm 27 \dagger^{* *}$ \\
& &
\end{tabular}

* Right side significantly different from left $(p<0.05$, Tukey's HSD test). ** Significantly different from untreated control $(p<0.05$, Tukey's HSD test). $\dagger$ Significantly different from vehicle group ( $p<0.05$, Tukey's HSD test). 

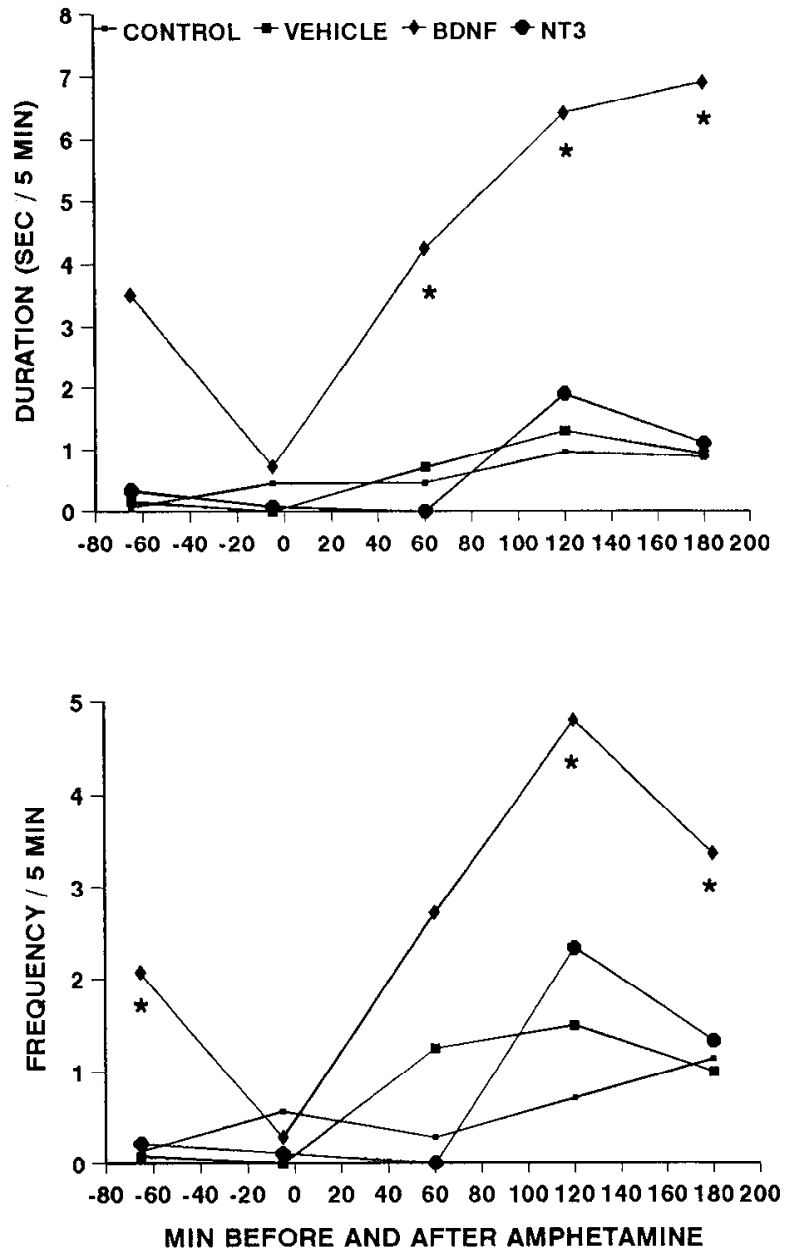

Figure 7. Mean duration (seconds per 5 min interval) and frequency (number in $5 \mathrm{~min}$ ) of amphetamine-induced backward walking for controls and for vehicle-treated, BDNF-treated, and NT-3-treated rats. *, significantly different from controls and vehicle infused rats $(p<0.05$, multiple $F$ test).

DA and 5-HT turnover in the right striatum, ipsilateral to the site of infusion.

\section{Discussion}

The present results clearly indicate that unilateral infusions of either BDNF or NT-3 immediately above the right SN alter circadian rhythms of locomotor activity, reduce body weight, elevate DA and 5-HT metabolism in the striatum, and decrease nigral DA metabolism. These diverse in vivo effects of BDNF and NT-3 are consistent with the ability of either protein to potentiate DA neuronal functions in vitro (Hyman et al., 1991; Knüsel et al., 1991) or in vivo for BDNF (Altar et al., 1992). BDNF and NT-3 preferentially bind to the TrkB and TrkC neurotrophin receptors, respectively (Lamballe et al., 1991; Soppet et al., 1991; Squinto et al., 1991; Altar et al., 1993). However, at higher concentrations, NT-3 can also activate the TrkB receptor whereas BDNF has very little affinity for the TrkC receptor. The greater behavioral and neurochemical effects of BDNF suggest that these effects are mediated by the TrkB neurotrophin receptor. This observation is consistent with the presence of BDNF and NT-3 mRNA (Gall et al., 1992), BDNFdisplaceable binding (Altar et al., 1993), and retrograde trans-
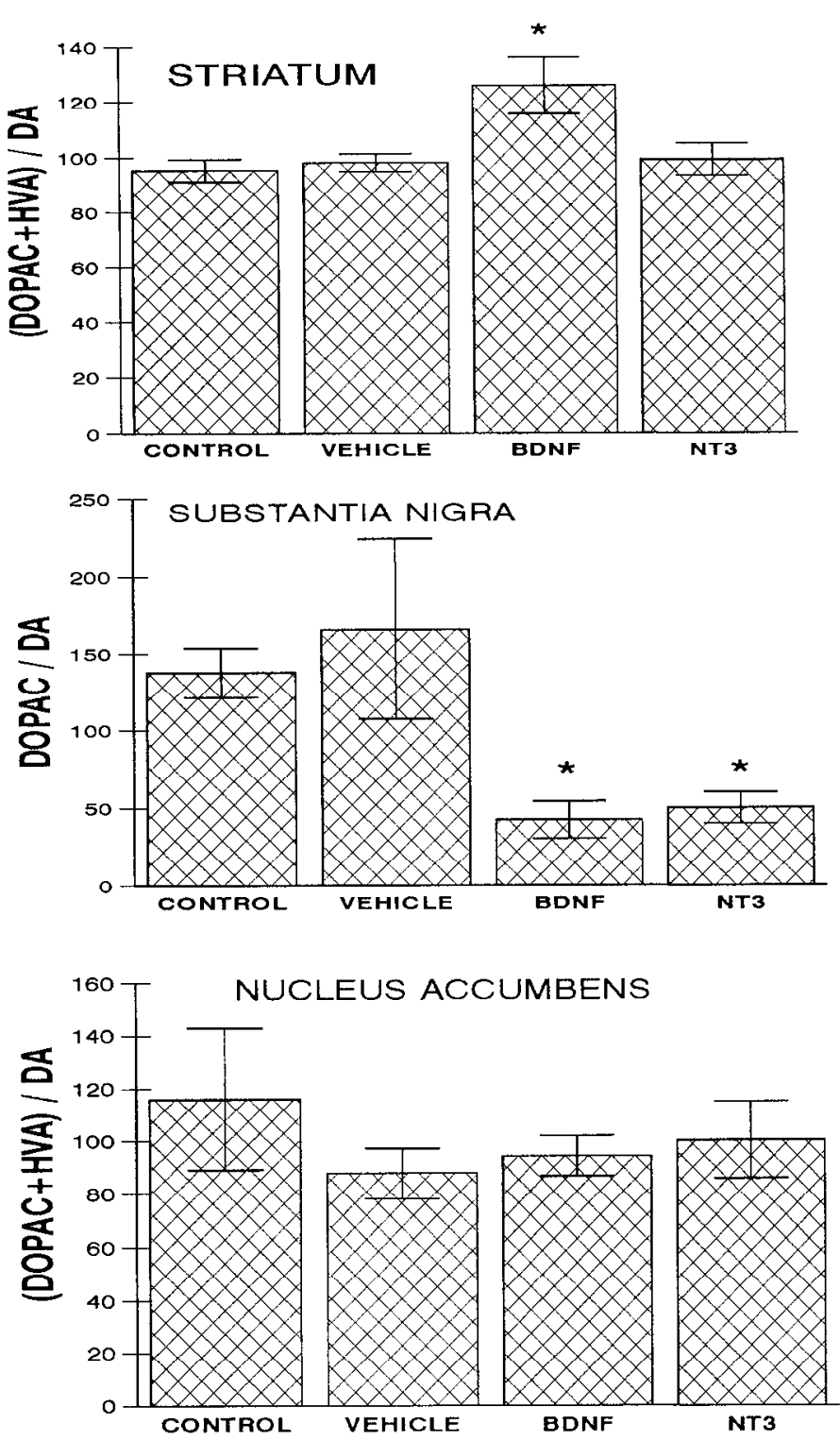

Figure 8. DA turnover in the striatum, SN, and nucleus accumbens for control and for vehicle-treated, BDNF-treated, and NT-3-treated groups. Values were obtained by the ratio (DOPAC + HVA):DA for the right striatum and nucleus accumbens, and DOPAC:DA for right SN (HVA levels were undetectable in this region), as a percentage of the same ratios from the left brain region. ${ }^{*}$, significantly different from both control and vehicle ( $p<0.05$, multiple $F$ test).

port of ${ }^{125}$ I-BDNF in the pars compacta of the SN (Wiegand et al., 1991).

Neurotrophins and body weight. The finding that both BDNF and NT-3 infused continuously and unilaterally into the SN reduces the body weights of rats supports a previous report that similar BDNF infusions into the SN (Altar et al., 1992) or lateral ventricle infusions of NGF (Williams, 1991) reduce body weight and food intake in rats. It is notable that no such reduction in body weights occurred for the vehicle-infused rats given free access to food, and that the amount of food available to these rats had to be restricted to maintain their body weights at levels similar to rats receiving BDNF and NT-3. Only one of four potential mechanisms that we have considered appears to explain these decreases in body weight. First, a potentiation of monoamine neuron activity (Blundell, 1984) may have been 
Table 3. Correlations between striatal neurochemistry and backward walking from the 5 min interval immediately prior to death of all rats from all treatment groups

\begin{tabular}{|c|c|c|c|c|}
\hline Behavior & $\begin{array}{l}\text { Striatal } \\
\text { side }\end{array}$ & $\begin{array}{l}\text { Neurochemical } \\
\text { measure }\end{array}$ & $\begin{array}{l}\text { Correlation } \\
\text { coefficient }\end{array}$ & $\begin{array}{l}\text { Signifi- } \\
\text { cance } \\
\text { level } \\
(p<)\end{array}$ \\
\hline \multirow{3}{*}{$\begin{array}{l}\text { Duration of } \\
\text { backward walking }\end{array}$} & \multirow[t]{3}{*}{ Right } & DOPAC & 0.32 & 0.05 \\
\hline & & Dopamine turnover & 0.38 & 0.02 \\
\hline & & 5-HT turnover & 0.31 & 0.05 \\
\hline \multirow{4}{*}{$\begin{array}{l}\text { Frequency of } \\
\text { backward walking }\end{array}$} & \multirow[t]{3}{*}{ Right } & DOPAC & 0.42 & 0.01 \\
\hline & & Dopamine turnover & 0.52 & 0.0005 \\
\hline & & 5-HT turnover & 0.40 & 0.01 \\
\hline & Left & DOPAC & 0.31 & 0.05 \\
\hline
\end{tabular}

responsible for the weight loss, as a result of a persistent amphetamine-like anorective effect. Howcver, rats habituate rapidly to the anorectic effects of amphetamine (Caul et al., 1988), whereas decreases in body weight (present study) and food intake (M. T. Martin-Iverson, K. G. Todd, and C. A. Altar, unpublished observations) persist for at least 2 weeks. Second, elevated striatal DA metabolism may have contributed to anorexia. However, DA metabolism was elevated only with supranigral infusions of BDNF (Altar et al., 1992) but not by NT-3 (Fig. 8) whereas both factors lowered body weight. Third, increases in locomotion could also have contributed to the decreases in body weight of the BDNF-treated rats due to increased energy demands. However, NT-3-treated rats lost the most body weight but showed no change in daytime locomotion and a decrease in nocturnal locomotion. Finally, the loss of body weights in BDNF-treated and NT-3-treated groups may have been due to increases in 5-HT turnover, since bilateral increases in striatal 5-HIAA were produced by factors both after amphetamine (present results) and during amphetamine-free periods when food intake is typically decreased (Altar et al., unpublished observations). Chronic treatment of rats with drugs that promote serotonergic functions, such as the 5-HT reuptake blockers fluoxetine and fenfluramine, decreases food intake and body weights in a sustained manner (Blundell, 1984; Carlton and Rowland, 1989).

Rotational and neurochemical effects of BDNF and NT-3. Both BDNF and NT-3 induced contraversive rotations after amphetamine, as has been previously reported for BDNF after a twofold higher dose of amphetamine (Altar et al., 1992). Two lines of evidence indicate that the ability of BDNF and NT-3 to alter spontaneous or amphetamine-stimulated behavior was probably not a consequence of the reduction in body weights produced by these compounds. First, reducing the weights of the vehicle-infused rats to match those of the treated groups did not decrease locomotion or induce net contraversive turnings after amphetamine. Second, the effects of BDNF and NT-3 on spontaneous locomotor activity and backward walking differed, although both neurotrophic factors had similar effects on body weight. It is also unlikely that the effects of BDNF on DA turnover were secondary to weight reduction, since weight reduction of the food-deprived vehicle group did not produce any measured alteration in DA or 5-HT neurochemistry compared to untreated animals.

Rotational behavior in rats with unilateral DA neuron lesions can result from an asymmetric activation of several neuronal systems. These include a relatively greater stimulation of postsynaptic DA receptors in the striatum contralateral to the direction of turning, due either to increased DA release, or to increased sensitivity or density of postsynaptic receptors (Ungerstedt and Arbuthnott, 1970). Alternatively, asymmetric stimulation by GABA agonists (Waddington, 1979), 5-HT agonists (Gerber et al., 1988), or dynorphin A (Matsumoto et al., 1988) can also induce contralateral rotational behavior, even in the absence of the DA neurons in the infused hemisphere. Thus, BDNF and NT-3 may enhance the rotational response to amphetamine by promoting activity of the infused DA neurons themselves, indicating a presynaptic locus of effect, or by postsynaptic means, via an elevated sensitivity of $D_{1}$ or $D_{2}$ receptors on the targets of DA release, or by effects on GABA, 5-HT, opioid, or other output systems. The former conclusion is supported by the finding that the net contraversive rotations correlated with decreases in the DA content of the right striatum, presumably as a result of increased DA release (Altar et al., 1992; present results). The present study also shows that BDNF or NT-3 each decreased DA turnover in the SN of the infused hemisphere, and this observation too supports a presynaptic locus of effect for the two neurotrophins. DA in the $\mathrm{SN}$ is released by the dendrites of activated DA neurons as part of a negative feedback regulatory system (Aghajanian and Bunney, 1977; Cheramy et al., 1981; Clark and Chiodo, 1988). Although BDNF and NT-3 induced contraversive rotations after amphetamine injections, only BDNF selectively increased DA turnover in the ipsilateral striatum. The neurochemical measures were obtained at $4 \mathrm{hr}$ after administration of amphetamine, when its behavioral effects were mostly absent. It remains possible that NT-3 increased DA turnover at a more behaviorally relevant time point. It is also pertinent that unilateral electrical stimulation of the medial forebrain bundle induces contraversive turning and increases bilateral 5-HT turnover in the striatum (Szostak et al., 1986) as seen here with BDNF and NT-3. The observation that extensive DA depletions, not obtained in the present experiment, are required to induce postsynaptic DA receptor supersensitivity (Neve et al., 1984) and the inability of apomorphine to induce contralateral rotations in rats with nigral infusions of BDNF (Altar et al., 1992 ) argues against increases in $D_{1}$-like or $D_{2}$-like receptors in causing the rotational effects of BDNF and NT-3. Thus, while other neuromodulatory or nigral output systems may be involved, the rotational behavior and neurochemical evidence is consistent with neurotrophin effects via DA and 5-HT neurons. 
Circadian rhythms in behavior. Compared to untreated controls, animals infused with vehicle displayed reduced levels of nocturnal locomotor activity and a phase shift of the acrophase (time of peak circadian activity rhythm). It seems unlikely that the motor activity effects in the vehicle-infused rats was due to their food restriction regimen since the reduction in locomotor activity occurred before food restriction. Furthermore, food restriction produced a transient increase in nocturnal locomotion that habituated after about three nights, and had no effect on locomotion during the daytime, when rats do not normally eat. Thus, food restriction would not be expected to decrease nocturnal locomotion. In addition, it is unlikely that the acrophase shift was due to a decrease in body weight, since an acrophase shift was not observed in the BDNF-treated group that showed similar decreases in body weights. The mechanism of the phaseshift induced by supranigral vehicle infusions is not presently known as no experiments have investigated the role of the SN in the timing of circadian rhythms. The behavioral effects of continuous vehiclc infusions into the $\mathrm{SN}$ may have been a function of damage produced by this procedure. Vehicle infusions above the $\mathrm{SN}$ produce an increase in ipsiversive rotations after amphetamine in about half the animals tested (Altar et al., 1992; present results), which would be consistent with damage to the DA system. However, nonpathological changes in physiology cannot be ruled out.

Other behavioral effects of BDNF and NT-3. The effect of NT-3 on locomotion was similar in some respects to the effects noted with vehicle infusions, which suggests that some of the effects were due primarily to the infusion procedure and not to the trophic factor itself. However, even when compared with vehicle, there was a greatly reduced amplitude of the circadian rhythm, best seen in the periodogram (Fig. 3). Amphetamine and a selective agonist for $D A D_{2}$ receptors can each increase the amplitudes of the peak diurnal locomotor rhythm (MartinIverson and Iversen, 1989; Yamada and Martin-Iverson, 1991; Martin-Iverson and Yamada, 1992), while widespread depletions of brain 5-HT decrease motor activity amplitudes in rats (Ohi et al., 1988). Thus, none of the observed postamphetamine neurochemical effects of NT-3 are consistent with its amplitudereducing actions. BDNF had very different effects on locomotor activity than either NT-3 or vehicle. It appeared to "normalize" the effects of vehicle, producing effects that were not different from untreated controls after the first $24 \mathrm{hr}$ of treatment. This may have resulted from a reversal of the consequences of damage produced by the treatment procedure, or reflect an indirect compensation produced by actions on some independent physiological process in the $\mathrm{SN}$, such as elevations in DA turnover (Altar et al., 1992).

Of all the other behaviors rated, only backward walking was significantly increased by BDNF, and this action was augmented by amphetamine. Turnover of both DA and 5-HT in the striatum correlated with backward walking, supporting a previous report that increases in the activity of both of these neurotransmitters provide central control over this behavior (Dourish, 1984).

While BDNF stimulated backward walking, and both BDNF and NT-3 induced amphetamine-stimulated contraversive turning, amphetamine-induccd locomotion was decreased by BDNF and NT-3, and other measures of amphetamine stereotypies, such as sniffing and head movements, were unaffected by the trophic factors. The fact that behaviors (locomotion, sniffing, rearing) associated with mesolimbic DA projection ar- eas (Costall et al., 1977; Morelli et al., 1980; Carey, 1983; Kelley et al., 1989) were largely unaffected or reduced by BDNF or NT-3, whilc rotational responses were augmented, is consistent with a selective augmentation by the infusions on mesostriatal and not mesolimbic DA function. In support of this conclusion, our infusions of either factor augmented mesostriatal but not mesolimbic DA turnover. It is likely that the chronic delivery of either factor above the pars compacta of the SN produced nigrostriatal and not mesoaccumbens changes. The $1.5-3 \mathrm{~mm}$ diffusion of BDNF or NT-3 following intraparenchymal infusion (Wiegand et al., 1991) would contribute to such an anatomically discrete effect. Indeed, an activation of monoamine systems in the nucleus accumbens and their behavioral output can be obtained with neurotrophin infusions into mesolimbic regions (Altar, unpublished observations).

Increasing the dose of amphetamine beyond a certain level can actually reduce locomotion while increasing oral stereotypies. This is sometimes explained by a response competition between incompatible behaviors (i.e., licking or gnawing part of a grid floor is incompatible with rotation, locomotion, and rearing). Response competition is not an adequate explanation of the present findings because neither the increases in rotation nor backward walking occurred with sufficient duration to compete with locomotion. Stereotyped behaviors, which could also compete for time spent locomoting, were unaffected by either neurotrophic factor. A more plausible explanation is that the increased 5-HT turnover produced by the trophic factors is responsible for the decreased amphetamine-induced locomotion. This interpretation is supported by the observation that depletions of 5-HT in the forebrain increase amphetamine-induced locomotion (Martin-Iverson et al., 1983).

Conclusions. In conclusion, the present data show for the first time that NT-3 and, to a greater extent, BDNF augment behavioral and neurochemical activities of the basal ganglia. Both factors altered neuronal DA and 5-HT turnover in a manner consistent with the decreases in body weight, increases in backward walking, and amphetamine-induced contraversive rotation. Thus, increases in DA turnover within the neostriatum obtained with BDNF, and decreases in DA turnover in the SN obtained with BDNF and NT-3 reflect an activation of mesostriatal DA pathways (Aghajanian and Bunney, 1977; Nieullion et al., 1979). Bilateral increases in striatal 5-HT turnover were obtained with either factor, and although the mechanism for these increases is unknown, these changes could act in concert with the increases in DA metabolism to alter circadian rhythms, basal locomotion, or other behaviors. The generally smaller behavioral and neurochemical effects of NT-3 are one example of how closely related members of the neurotrophin family can display considerably different in vivo actions. Thus, while BDNF and NT-3 have a similar pattern of effects on some measures, differences in their behavioral effects may be due to their preferential activation of different neurotrophin receptors. On the other hand, in the absence of a dose-response relationship for either BDNF and NT-3, it is possible that the different behavioral effects may be due to a lack of equivalence between the same doses of the two neurotrophins used in the present study

\section{References}

Aghajanian GK, Bunney BS (1977) Dopamine autoreceptors: pharmacological characterization by microiontophoretic single cell recording studies. Naunyn Schmiedebergs Arch Pharmacol 297:1-8.

Alderson RF, Alderman AL, Barde Y-A, Lindsay RM (1990) Brain 
derived neurotrophic factor increases survival and differentiated functions of rat septal cholinergic neurons in culture. Neuron 5:297-306.

Altar CA, Boylan CB, Jackson C, Hershenson S, Miller G, Wiegand SJ, Lindsay RM, Hyman C (1992) Brain-derived neurotrophic factor augments rotational behavior and nigrostriatal dopamine turnover in vivo. Proc Natl Acad Sci USA 89:11347-11351.

Altar CA, Criden MR, Lindsay RM, DiStefano PS (1993) Characterization and topography of high affinity ${ }^{125} \mathrm{I}$-neurotrophin-3 binding to mammalian brain. J Neurosci 13:733-743.

Baker GB, Coutts RT, Rao TS (1987) Neuropharmacological and neurochemical properties of $N$-(2-cyanoethyl)-2-phenylethylamine, a prodrug of 2-phenylethylamine. Br J Pharmacol 92:243-255.

Blundell JE (1984) Serotonin and appetite. Neuropharmacology 23: 1537-1551.

Carey RJ (1983) Differential effects of limbic versus striatal dopamine loss on motoric function. Behav Brain Res 7:283-296.

Carlton J, Rowland NE (1989) Long term actions of $d$-fenfluramine in two rat models of obesity. I. Sustained reductions in body weight and adiposity without depletion of brain serotonin. Int J Obesity 13: $8325-8347$

Caul WFW, Jones JR, Barrett RJ (1988) Amphetamine's effects on food consumption and weight: the role of adaptive processes. Behav Neurosci 102:441-450.

Cheramy A, Leviel V, Glowinski J (1981) Dendritic release of dopamine in the substantia nigra. Nature 289:537-542.

Clark D, Chiodo LA (1988) Electrophysiological and pharmacological characterization of identified nigrostriatal and meso-accumbens dopamine neurons in the rat. Synapse 2:474-485.

Costall B, Marsden CD, Naylor RJ, Pycock CJ (1977) Stereotyped behaviour patterns and hyperactivity induced by amphetamine and apomorphine after discrete 6-hydroxydopamine lesions of extrapyramidal and mcsolimbic nuclci. Brain Res 123:89-111.

Dourish CT (1984) Studies on the mechanism of action of $\beta$-phenylethylamine animal model of schizophrenia. In: Neurobiology of the trace amines (Boulton AA, Baker GB, Dewhurst WG, Sandler M, eds), pp 389-411. Clifton, NJ: Humana.

Gall CM, Gold SJ, Isackson PJ, Seroogy KB (1992) Brain-derived neurotrophic factor and neurotrophin-3 mRNAs are expressed in ventral midbrain regions containing dopaminergic neurons. Mol Cell Neurosci 3:56-63.

Gerber R, Altar CA, Liebman JM (1988) Rotational behavior induced by 8-hydroxy-DPAT, a putative 5HT-1A agonist, in 6-hydroxydopamine-lesioned rats. Psychopharmacology 94:178-182.

Halberg F, Johnson EA, Nelson W, Runge W, Sothern RB (1972) Autorhythmometry procedures for physiological self measurements and thcir analysis. Physiol Teach 1:1-11.

Hartikka J, Hefta F (1988) Function of neurotrophic factors in the adult and aging brain and their possible use in the treatment of neurodegenerative diseases. J Neurosci 8:2967-2985.

Hefti $F$ (1986) Nerve growth factor promotes survival of septal cholinergic neurons after fimbrial transections. J Neurosci 6:2155-2162

Hyman C, Hofer M, Barde Y-A, Jurhasz M, Yanacoupolos G, Squinto SP, Lindsay RM (1991) BDNF is a neurotrophic factor for dopaminergic neurons of the substantia nigra. Nature 350:230-232.

Kelley AE, Gauthier AM, Lang CG (1989) Amphetamine microinjections into distinct striatal subregions cause dissociable effects on motor and ingestive behavior. Behav Brain Res 35:27-39.

Kiess HO (1989) Statistical concepts for the behavioral sciences. Toronto: Allyn and Bacon.

Knüsel B, Winslow FW, Rosenthal A, Burton LE, Seid DP, Nikolics K, Hefti F (1991) Promotion of central cholinergic and dopaminergic neuron differentiation by brain-derived neurotrophic factor but not neurotrophin-3. Proc Natl Acad Sci USA 88:961-965.

Lamballe F, Klein R, Barbacid M (1991) trkC, a new member of the trk family of tyrosine protein kinases, is a receptor for neurotrophin3. Cell 66:967-979.

Leibrock J, Lottspeich F, Hohn A, Hofer M, Masiakowski P, Thoenen $\mathrm{H}$, Barde Y-A (1989) Molecular cloning and expression of brain derived neurotrophic factor. Nature 341:149-152.

Lindsay RM, Barde Y-A, Davies AM, Rohrer H (1985) Differences and similarities in the neurotrophic requirements of sensory neurons derived from neural crest and neural placode. J Cell Sci [Suppl] 3:1 15129.

Maisonpierre PC, Belluscio L, Squinto S, Ip NY, Furth ME, Lindsay RM, Yancopoulos GD (1990) Neurotrophin-3: a neurotrophic factor related to NGF and BDNF. Science 247:1446-1451.

Martin-Iverson MT (1991) An animal model of stimulant psychoses. In: Neuromethods: animal models in psychiatry I, Vol 18 (Boulton AA, Baker GB, Martin-Iverson MT, eds), pp 103-149.

Martin-Iverson MT, Iversen SD (1989) Day and night locomotor activity effects during administration of $(+)$-amphetamine. Pharmacol Biochem Behav 34:465-471.

Martin-Iverson MT, Yamada N (1992) Synergistic effects of dopamine D1 and D2 receptor agonists are determined by circadian rhythms. Eur J Pharmacol 215:119-125.

Martin-Iverson MT, LeClere J-F, Fibiger HC (1983) Cholincrgic-dopaminergic interactions and the mechanisms of action of antidepressants. Eur J Pharmacol 94:193-201.

Matsumoto RL, Brinsfield KH, Patrick RL, Walker JM (1988) Rotational behavior mediated by dopaminergic and non-dopaminergic mechanisms after intranigral microinjection of specific mu, delta and kappa opioid agonists. J Pharmacol Exp Ther 246:196-203.

Morelli M, Porceddu ML, Di Chiara G (1980) Lesions of substantia nigra by kainic acid: effects on apomorphine-induced stereotyped behaviour. Brain Res 191:67-78.

Neve KA, Altar CA, Wong CA, Marshall JF (1984) Quantitative analysis of $\left[{ }^{3} \mathrm{H}\right]$ spiroperidol binding to rat forebrain sections; plasticity of neostriatal dopamine receptors after nigrostriatal injury. Brain Res 302:9-18.

Ohi K, Hayashi S, Takahashi K (1988) Development of circadian rhythms in rats with lesions of serotonergic system. Physiol Behav 44:393-403.

Rosenthal A, Goeddel DV, Hguyen T, Lewis M, Shih A, Laramee GR, Nikolics K, Winslow JW (1990) Primary structure and biological activity of a novel human neurotrophic factor. Neuron 4:767-773.

Soppet D, Escandon E, Maragos J, Middlemas DS, Reid SW, Blair J, Burton LE, Stanton BR, Kaplan DR, Hunter T, Nikolics K, Parada LF (1991) The neurotrophic factors brain-derived neurotrophic factor and neurotrophin-3 are ligands for the trkB tyrosine kinase receptor. Cell 65:895-903.

Spina MB, Squinto SP, Miller J, I indsay R, Hyman C. (1992) Brainderived neurotrophic factors protects dopamine neurons against 6-hydroxydopamine and $N$-methyl-4-phenylpyridinium ion toxicity: involvement of the glutathione system. J Neurochem 59:99-106.

Squinto SP, Stitt T, Aldrich TH, Davis S, Bianco SM, Radziejewski C, Glass DJ, Masiakowski P, Furth ME, Valenzuela DM, DiStefano PS, Yancopoulos GD (1991) irkB Encodes a functional receptor for brain-derived neurotrophic factor and neurotrophin-3 but not nerve growth factor. Cell 65:885-893.

Szostak C, Jakubovic A, Phillips AG, Fibiger HC (1986) Dopaminergic and serotonergic correlates of stimulation-induced circling. Behav Brain Res 21:37-46.

Ungerstedt $U$ (1971) Striatal dopamine release after amphetamine or nerve degeneration revealed by rotational behaviour. Acta Physiol Scand 83[Suppl 367]:49-68.

Ungerstedt U, Arbuthnott GW (1970) Quantitative recording of rotational behavior in rats after 6-hydroxydopamine lesions of the nigro-striatal dopamine systems. Brain Res 24:485 493.

Vitaliano PP (1982) Parametric statistical analysis of repeated measures experiments. Psychoneuroendocrinology 7:3-13.

Waddington JL (1979) A methodological weakness in the use of neuroleptic antagonism as a sole criterion for DAergic mediation of druginduced behavioral effects. Eur J Pharmacol 58:327-329.

Wiegand SJ, Alexander C, Lindsay RM, DiStefano PS (1991) Axonal transport of $\left[{ }^{125} \mathrm{I}\right]$-labeled neurotrophins in the central nervous system. Soc Neurosci Abstr 17:1121.

Williams LR (1991) Hyperphagia is induced by intracerebroventricular administration of nerve growth factor. Exp Neurol 113:31-37.

Yamada N, Martin-Iverson MT (1991) Selective dopamine D1 and D2 agonists independently affect different components of the freerunning rhythm of locomotor activity in rats. Brain Res 538:310312. 